\title{
The experience of recovering gamblers in Malaysia: a phenomenological study
}

\author{
Li Li Choong ${ }^{1}$, Jasmine M Y Loo ${ }^{2^{*}}$ and Wai Sheng $\mathrm{Ng}^{3}$
}

\author{
* Correspondence: jasmine.loo@ \\ monash.edu \\ 2Jeffrey Cheah School of Medicine \\ and Health Sciences, Monash \\ University Malaysia, Jalan Lagoon \\ Selatan, Bandar Sunway 47500, \\ Malaysia \\ Full list of author information is \\ available at the end of the article
}

\begin{abstract}
Understanding the problem gambling recovery process is essential in the development of effective prevention and treatment programs. Existing empirical studies have examined the determinants and treatment of gambling behaviour but little research has investigated the experiences of Asian gamblers and their perceptions of gambling impact on family and significant others. This study utilizes a phenomenological approach to examine the experiences of ten gamblers who were enrolled in a gambling recovery program. Data analyses on qualitative interview sessions revealed four main themes comprising meaning of gambling, beliefs about gambling, perception of themselves (gamblers), family and significant others, and factors associated with the cessation of gambling behaviour. Within each theme, there are interrelated sub-themes that will be discussed within the study. In sum, the common factors that were evident are the importance of the gambler's motivation to quit gambling and also their family's support in the recovery process. Suggestions and implications for treatment and recovery are discussed.
\end{abstract}

Keywords: Recovery; Problem gambling; Asian; Family and significant others

\section{Background}

Gambling is a behaviour where individuals wager monetary possessions on events that are determined by probability or chance (Lau and Ranyard, 2005; Raylu et al., 2008). Definitions of gambling, factors that affect the development of pathological gambling and cognitive behavioural viewpoints of gambling have predominantly been areas of research interest (Loo et al., 2012; Raylu et al. 2013). However, there seems to be a lack of research on problem gamblers' recovery experience, perceived importance of families during recovery and the meaning of gambling. Research results have often focused on the characteristics of gamblers (American Psychiatric Association, 2000; Ciarrocchi and Richardson, 1989) and the possible cognitive structure of a gambler's perceptions (Rossi, 1980). However, past research lacks emphasis on the meaning of gambling to Asian problem gamblers and factors associated with their turning-point and decision to quit gambling.

Exploration of the meaning of gambling to gamblers and their life experiences will enhance our understanding of the problem gambling recovery process. Connections between factors contributing to the recovery process to the lives of gamblers can be further expanded through the means of phenomenological studies, as individuals who have gone through the same experience may have different interpretations of that

(c) 2014 Choong et al.; licensee Springer. This is an open access article distributed under the terms of the Creative Commons Attribution License (http://creativecommons.org/licenses/by/2.0), which permits unrestricted use, distribution, and reproduction in any medium, provided the original work is properly cited. 
experience. Phenomenological studies are concerned with the perspective of each individual and their perceived experience while going through a determined event. These individual differences in gambling behaviour and recovery experiences are our main focus in this study. We identify gaps in our understanding with information provided by past research that are further explored in this study. The review of literature focuses on the definition of gambling and the experience of gamblers, their family and significant others.

\section{Definition of gambling}

Gambling behaviour is a widespread occurrence that functions as an entertainment and leisure activity that has devastating consequences if one is preoccupied with the behaviour. Gambling is often viewed as a social activity that is precipitated by an urge to seek pleasure or excitement. However, social gambling may escalate to problem gambling if the behaviour persists. In addition, being addicted means that one will be psychologically and compulsively involved in it and addicted gamblers can lose control over their ability to withhold themselves (Scanlon and Whitehead, 2004). There are many people who gamble for fun or for recreational purposes, which starts with the innocent thought of testing their luck by betting in small amounts. However, some individuals grow to become gripped by this game of chance to the point that they are no longer able to control their gambling urges.

Therefore, understanding gambling precipitators is very important because it is a threshold to problem and pathological gambling from recreational gambling. Pathological gambling can be defined as a persistent and recurrent maladaptive gambling behaviour that meets the criteria in DSM-IV-TR (American Psychiatric Association, 2000; Loo et al., 2008; Raylu and Oei, 2002). It is categorized as part of "Addiction and Related Disorders" in the updated DSM-5 (American Psychiatric Association, 2013) and has a lifetime prevalence ranging from $0.4 \%$ to $3.4 \%$ in adults and $2.8 \%$ to $8 \%$ for adolescents and college students in prevalence research in Hong Kong, Scotland, United States and Britain (Kim et al., 2008; Moodie and Finnigan, 2006; Volberg et al., 2006; Wardle et al., 2007). Access to gambling and the length of access influences the prevalence of pathological gambling; therefore, an increase in access such as legalized gambling may potentially lead to increased prevalence.

On the other hand, individuals who do not meet the diagnostic criteria of pathological gambling in DSM-IV-TR but struggle with gambling-related issues are typically referred to as "problem gamblers" (PG) in the literature (Clarke et al., 2006; Loo et al., 2008). Individuals struggling with problem gambling often cannot control gambling urges and are habitually preoccupied with thoughts of gambling (Turner, 2008; Wolfling et al., 2011). They will spend a considerable amount of time and money to fuel their disruptive habit even to the extent of risking their finances and health conditions (Schwarz and Lindner, 1992; Teo et al., 2007). This disordered pattern of behaviour can lead them into huge financial debts and strained relationships with their family and significant others (Basu, 1991; Oei et al., 2008; Vong, 2007).

Furthermore, it has been argued that gamblers go through a series of stages before evolving into pathological gamblers (Nixon and Solowoniuk, 2006). An individual normally starts off by gambling for enjoyment and social purposes. Subsequently, when the individual starts to win and when self-esteem is associated with success, the 
chances of progressing to the next stage may advance as wins reinforce gambling behaviour associated with positive emotions. Finally, if the individual encounters a big win rewarded with a large amount of money, then the gambling urge to re-experience that positive emotional event will be very strong (Raylu and Oei, 2004a). This may lead to a situation where the individual will be caught in a cycle of trying to relive the experience and constantly motivated to achieve that experience of winning (Lee et al., 2007).

In addition, Blaszczynski and Nower (2002) have suggested that the development of pathological gambling does not necessary follows a single pathway or theoretical model of gambling. They proposed that pathological gamblers may be influenced differently through biological, psychological or ecological factors. They identified three distinct subgroups of pathological gamblers. The first group is argued to be behaviourally conditioned through different learning processes, the second group is emotionally vulnerable and uses gambling as a coping mechanism, and the third group of gamblers have antisocial and impulsivity tendencies (Blaszczynski and Nower, 2002).

\section{Experience of gamblers, their family and significant others}

Past research suggests that gambling may affect the relationship between the gambler and their family or significant others. A qualitative study conducted by Dickson-Swift et al. (2005) found that excessive gambling affected several aspects of a couple's life where one spouse was gambling. Financial instability, strained family relationships, poor physical health and disturbed emotional well-being are some of the negative impacts of gambling on gamblers' spouses and family members (Dickson-Swift et al., 2005).

In addition, Valentine and Hughes (2010) discovered how online gamblers' significant others managed the disclosure of the gambler's habit through their supportive reaction and behaviours. Their study indicated that family support is important in the recovery process and suggested strategies of recovery that were defined within the family context. Some participants (recovering gamblers) did not agree with the way their family solved the issue by helping to pay off their gambling debts. However, recovering gamblers abstained longer when their significant others (spouse, siblings, parents or children) restricted their finances and provided support for their behavioural change (i.e., gambling cessation).

Moreover, Corney and Davis (2010) found that the relationship between female internet gamblers and their significant others (husband, children or family members) were strained due to deceptions used while concealing their gambling habit and lack of time spent with their family. Furthermore, adverse financial consequences were reported. However, they received both emotional and physical support from significant others after the disclosure of their gambling habit. Some gamblers indicated that they preferred significant others to take control and disapprove of their gambling behaviour in order to facilitate the process of gambling cessation (Corney and Davis 2010).

\section{Factors and motivations associated with the development of pathological gambling}

Past research suggests there are several factors associated with the development of problem and pathological gambling. According to Wolfling et al. (2011), the psychophysiological approach looks at how stimuli related to gambling evoke the attention of 
pathological gamblers as compared to non-gamblers. Pathological gamblers are also more likely to crave for gambling-related stimuli as compared to neutral stimuli. This research suggests that craving and relapse of pathological gamblers can be measured biologically. In addition, Bagby and colleagues (Bagby et al., 2007) elaborated on certain personality factors and individual differences, such as impulsivity, antisocial behaviours, maladaptive coping strategies and the explanatory style of an individual, as risk factors that contribute to the development of pathological gambling.

Furthermore, socio-cultural factors play an important role in determining the start and maintenance of gambling behaviour. Living within certain cultures that encourage or endorse gambling as a recreational activity (e.g., Chinese and Koreans) is different as compared to living in a culture where gambling is illegal (e.g., Muslims under the Syariah law) or not a socially acceptable activity. For example, Lam (2008) wrote about the brief history of gambling within the Chinese community of China. Despite repeated bans and strict regulation by many ancient Chinese imperial rulers, gambling flourished to become a social activity and is considered favourably as the national pastime by most Chinese. Gambling is also seen as a method of gaining revenue with the motivation of wanting instantaneous financial gains.

In addition, the proliferation of public lottery, racetrack gambling, legalized casinos (Kim, 2011) and electronic gambling machines in China (Papineau, 2013) may contribute to increased problem gambling as gambling becomes a way-of-life within the Chinese community (Papineau, 2001). It is interesting to note that the Paichais of Macau (Chan and Ohtsuka, 2013) seemed to have created a sub-culture within the Macau gambling community. A qualitative study on Vietnamese Australian casino gamblers (Ohtsuka and Ohtsuka, 2010) found that, although Vietnamese Australians held similar universal beliefs about gambling as found in other cultures, there were certain culturespecific schemas that were present in their beliefs about gambling consequences. In short, these studies reinforce the impact of culture on gambling propensity, which further strengthens the rationale for conducting qualitative research on Asian gamblers' experiences.

Moreover, cognitive patterns of pathological gamblers can be transferred intergenerationally (Oei and Raylu, 2004) as values and information about gambling are transmitted across generations and parents' perception about gambling influences children's perception of gambling. Research on familial influences (Eisen et al., 1998) concluded that vulnerability in developing pathological gambling is substantially influenced by familial factors such as genetic and/or shared environment of twin siblings. Other evidence suggests that familial factors may contribute to the development and maintenance of pathological gambling (Ciarrocchi and Reinert, 1993) but past research only shows high prevalence between parents or relatives who gamble and the development of their children's gambling habit (Darbyshire et al., 2001; Raylu and Oei, 2004b). However, past research has not explored the role of family in gambling abstinence or the turning-point in which pathological gamblers choose to quit gambling.

\section{Current phenomenological research}

As stated earlier, gambling for many may just be a harmless leisure activity but a growing number of gamblers are starting to lose control of their habit and gamble compulsively (Thomas et al., 2009). In this study, we are interested in exploring the lived 
experience, meaning of gambling and family's involvement in the recovery process from the perspective of the gambler. This qualitative study aimed to address the following research questions:

- What is the meaning of gambling to recovering gamblers?

- What are the life experiences of recovering gamblers?

- What are the implications of family processes in gamblers' recovery progression?

Answers to these research questions will enhance our ability to meet the needs of recovering gamblers and provide practical implications for service providers.

Qualitative methodologies in gambling research provide important insights into the underlying mechanisms by allowing in-depth exploration of research data. These methods have been employed in different aspects of gambling research such as the impact of gambling on spousal relationships (Dickson-Swift et al., 2005), Indigenous Australian's gambling-related crime and potential public intervention (Breen et al., 2013) and helpseeking behaviour of recovering gamblers (Nuske and Hing, 2012). Qualitative phenomenological analyses explore concepts and provide additional insights that may not be captured adequately through quantitative research. As phenomenological studies investigate the essence and structure of an individual's experiences in certain situations, it focuses on how people view and perceive the world around them (Baker et al., 1992; Giorgi, 1989; Merriam, 2002). In this study, we attempt to understand the phenomenon from the participants' vantage point and identify similarities and differences between participants (Baker et al., 1992). Giorgi's (1989) analytical structure was used to analyse interview research data.

\section{Method}

Participants

Ten participants were recruited via purposive sampling for individual interview sessions. Participants were recruited from the Gamblers Rehabilitation Centre in Malaysia. Participants were males as this inpatient centre only admits primarily male gamblers of Chinese ethnicity. Outpatient programs cater to follow-up recovery and relapse prevention. Literacy was not compulsory because instructions were verbally communicated. Participants however, were required to be able to converse in English. In this centre, the age of participants ranged from 18 years to 57 years with an average age of 43 years. In addition, all participants had resided in the centre for more than three months in the recovery program. All participants interviewed were identified by the centre's facilitator. However, their voluntary consent was ensured by the researcher again during the interview via the explanatory statement to participants and consent forms. Participants were informed that they could terminate the interview session at any time if they felt uncomfortable.

\section{Materials}

An information pack was prepared to ensure that participants understood the nature of the study. This pack included a cover letter, explanatory statement, consent for participation, and consent for audio recording. A standardized script was prepared to facilitate the interview process and was used with all participants. An audio recorder was used to record the interview sessions. 


\section{Procedure}

This research was reviewed and approved by the Ethics Review Board of the Department of Psychology at the Faculty of Behavioural Sciences of HELP University, Malaysia. Permission for recruitment of participants was obtained from the Gamblers Rehabilitation Centre. The recorder was turned on with informed consent after the participants were comfortable and ready to start the interview process. The session was a semi-structured interview with several questions guiding the discovery of phenomenon (i.e., gambling behaviour). The opening statement posted to participants was "Please describe your perceptions of gambling." The participants were then probed to explore their answers further. The role of the interviewer was to listen and guide participants while they described their experiences to ensure that the essence of their experiences was associated with the phenomenon.

The sessions lasted between 35 minutes to an hour as each participant was given flexibility in time to explore the issues being discussed. Any ambiguity during the interview was clarified by asking participants to elaborate on the discussion. In addition, the interviewer focused on listening to the structure of the experience from the participants' viewpoint regarding their gambling experience. The interviewer ensured that the questions asked were free from experimenter bias and social desirability. Before ending the session, the participants were encouraged to verbalise any additional comments. Finally, they were thanked for their co-operation and contribution to the research.

\section{Data analysis}

Initially, the recorded interview sessions were transcribed verbatim into individual transcripts. The researcher referred to Giorgi's (1989) four basic steps to analyse the data. Analyses were carried out using a scientific phenomenological reduction method where only the experiences linked with the phenomenon were reduced. The process of analysis was divided into two parts where the first part was individual analysis and the second were group analyses.

\section{Individual analysis}

Giorgi (1989) and Merriam (2002) outlined the four steps as follows:

1. The process of analysis begins with the researcher listening to the interview and reading the entire description of the first script, and trying to sense the whole experience of the first participant.

2. The whole is then broken into smaller relevant parts that are also called meaning units. Meaning units are parts that are on a similar topic or issue. In this step, whenever the researcher feels that there is a shift of meaning in what is said by the participant, a mark is made at those shifts. A shift of meaning indicates that the participant started talking about a different topic or issue.

3. These meaning units are then transformed into psychological components that reflect the explicit content, implicit content and how they are said by the participant.

4. Later, the researcher synthesizes the meaning units and uses them to discuss the structure of experience.

The same coding process was repeated for all transcripts. 


\section{Group analyses}

At this step, extracted elements from the structure of experience of the participants were used to discuss the similarities and differences between data. This was to uncover some general descriptions of the phenomenon being studied, which is the experience of recovering gamblers (Giorgi, 1989). Group analyses were constructed after data from six participants were coded. Any similar or new descriptions of the subsequent transcripts were added into the existing structure of experience.

\section{Validity}

Three peer reviewers were engaged to ensure validity (one clinical psychologist and two third-year undergraduate psychology students). Peer reviewers were briefed about the nature of this study and the concept of phenomenological studies. They were also required to do bracketing, which was an attempt to put any of their pre-existing biases or underlying assumptions regarding the phenomenon (gambling) aside.

Peer review was conducted by discussing the formation of themes and ideas against the unprocessed data (firsthand interview scripts) plus the interpretation of those psychological themes. Peer review was done twice; first after data from six participants were coded and the second review after data from ten participants were coded. All of the analyses were done in the presence of the interviewer and peer reviewers in a group discussion. Members of the peer review panel were allowed to read the manually coded scripts of the interviewer and had the opportunity to question the interviewer about the formation of the meaning units and psychological themes that were written. Any discrepancies or suggestions were discussed. The advantages of using this strategy were that the researcher's biases and interpretation were verified by the peer reviewers and emerging ideas were discussed thoroughly.

\section{Results}

Analyses of data generated four themes of the whole phenomenon, which is the experience of recovering gamblers. Each structure holds different themes that are linked together and understood as a whole and not just a sum of its parts. Please refer to Figure 1 for the structures or themes. The themes are meaning of gambling, beliefs about gambling, gambler's self-perception and cessation of gambling. It is important to note that the themes: meaning of gambling and beliefs about gambling are distinct from one another. The meaning of gambling is described as the significance of the meaning of gambling behaviour to the participants while beliefs about gambling is described as the acceptance of a statement as true or real.

\section{Meaning of gambling}

Gambling starts off as a pleasurable activity to gamblers. Some participants viewed gambling as a pleasurable, fun (P5) and enjoyable activity (P4, P6 and P10). P1 even mentioned that gambling activities elicited excitement and meaning to his life. In addition, gambling was also associated with how the gamblers would like to obtain recognition and dignity from their families and friends (P1). Gambling activity and monetary revenues were seen as ways to gain respect from family and significant others. Financial gains represented an increase in the positive perception of social status among significant others that is linked with the gamblers' self-reported trust and self-esteem. 


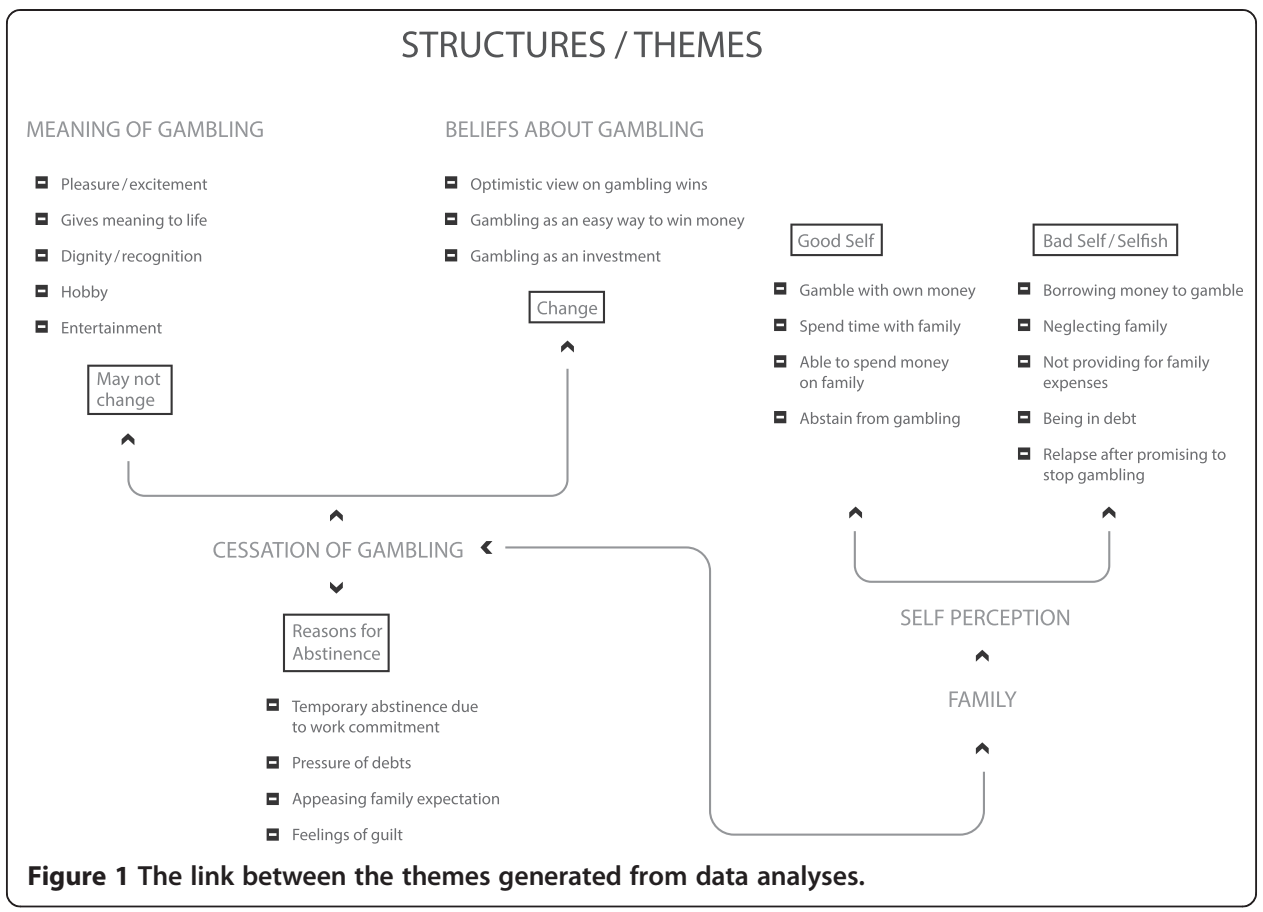

(P10) And we enjoy the whole process. We enjoy the [gambling] activity and once we go into gambling we [will] forget a lot of things.

(P1) This is the only way I can prove to my father that I can be much better than him because my father emphasizes on money a lot. So, I want to earn a lot of money in a fast manner [and that is] through gambling. All my relatives had graduated, found a good job with high salary so I want to prove to people that I am not losing out.

(P4) I kept on gambling not because I want to win back all the money but it is because I want to win back all the trust, self-esteem and good relationships. I have always thought that if I have money, I will have all these things back.

Furthermore, participants reported that gambling was also a hobby or a preferred pastime that is viewed concurrently as a source of entertainment (P2, P5, P7 and P9).

(P2) I gamble to fill up my time. So, it's just like a hobby.

(P5) Gambling initially was just only a source of entertainment where you go and have fun.

\section{Beliefs about gambling}

One belief that participants held onto was that they would someday win a large amount of money and gain significant financial revenues from "investing" in gambling activities. This belief precipitated their gambling behaviour. In addition, winning a large amount of money led participants to think that money comes easily through gambling-related activities. They viewed gambling as an investment and avenue to win easy money 
without working hard for it. For some, the belief about gambling as a game of chance serves as a foundation for the development of pathological gambling. Large wins earlier on in their gambling experiences further reinforce their beliefs about gaining revenues through gambling.

(P4) [40 years ago] My salary was only RM180 per month and by chance I can earn a lot of money through gambling. I won approximatelyRM7000, which is almost equivalent to my three-years salary. This attracted me a lot because I thought that instead of working hard for three years; I can earn such an amount through gambling.

(P5) I only gamble in the casino as I see it as just an investment to get monetary returns.

\section{Perceptions about themselves (gamblers)}

This theme emerged as the participants were describing their family experiences. Therefore, their perception of self is closely linked with how they perceive their families (e.g., familial needs and happiness) and how significant others perceive them (e.g., dissatisfaction expressed by the gambler's spouse regarding financial predicament). Some participants saw themselves as a good father or husband. Participants reported satisfaction when they were able to gamble with their own money (i.e., not borrowed), spend time with their family, provide for the family's needs and abstain from gambling.

(P5) And honestly when I am not gambling, I can consider myself as a good father or a good husband. After work I would go back and I don't gamble at night.

(P4) I am very happy when I win via gambling as I get to bring my family on holiday trips. Imagine having the option to go anywhere and enjoy spending the money by buying them their favourite foods and gifts.

On the other hand, the participants started to view themselves negatively or as being selfish when they started to borrow money to gamble and when they were in debt. This negative perception of themselves persists when they perceive that they have neglected their family, when they are not able to provide for their family financially, and when they relapse after promising their family that they will stop gambling. Participants reported developing a sense of hopelessness when they perceived that they have continuously disappointed their family members.

(P10) When I was gambling, I didn't provide a comfortable living environment to my wife and family. I'm not a good father. I never teach my children what is good and bad. I set a very bad example for them. I never watched movies with them. [Families] go on holidays and go shopping together but I do not do it as I'm always away from my family because of my gambling habit.

(P1) My family had helped me so many times [six to seven times already]. So, I do not dare let my family members know so I.. [took bank loans that I cannot afford and got blacklisted from these financial institutions]. 
(P3) Your family might forgive you a couple of times but eventually when you gamble non-stop, how are they going to forgive you?

\section{Cessation of gambling}

One of the recurring reasons for periodic gambling abstinence was that gamblers needed to spend their time working to earn more money to gamble and to pay off debts. In addition, some gamblers reported that they abstained from gambling to appease family members who helped pay off their debts. Moreover, they also felt guilt for putting their family through all the pain and inconvenience.

(P3) When I'm aware that my family is very unhappy with my gambling behaviour,

I'll stop for up to six months to a year and then I usually relapse back into gambling.

(P2) What led me to stop gambling? I was sitting there observing my family who were feeling very tired because of their long hours of work. My father cannot eat and sleep well. My heart aches. I can feel their pain. My heart was very touched and I think,

"That's it, [I will] quit gambling."

However, some participants decided to quit gambling only when their family members no longer offered assistance in solving their debt issues, and when their family severed relational ties or would not communicate with them anymore. The perception is that their family no longer has hope in them. Thus, this led them to join the Gamblers Rehabilitation Centre with hopes of recovering from their gambling habit.

(P7) When [my children] were younger, they did not understand much. Now they understand the situation as they are older. My daughter still does not talk to me. Initially, I was not very used to it so I try to go back home after joining the rehabilitation program. But my wife told me, "No, you cannot come back home, you have to go back [to the recovery program]."

(P9) To be honest, my wife and family paid my debts twice because my children were very young so they reconciled with me but now my children are all older. They have their own families now. [pause with sadness] I know that they are still very disappointed in me.

It must be noted that after participants decided to join the Gamblers Rehabilitation Centre, their family's support played a fundamental role in encouraging their recovery process (Kim, 2011; Loo et al., 2011). Most participants enrolled in the rehabilitation program with hopes of abstaining from gambling and eventually providing restitution for their families.

(P7) [I feel] totally different now as I do not need a lot of money in my wallet. In the past, I felt very uneasy when my wallet does not have at least a few hundred ringgits. Now, I just have RM2O in my wallet. My family accepted me back during recovery and I do spend more time with my wife and children now.

(P10) When I was still gambling, my children accepted me as their father because they were scared and afraid. Now they have seen me change and seen what I have 
done, I think they are more willing to accept me into their lives. I feel they are accepting me now.

\section{Discussion}

Four themes were constructed from analysing the phenomenon under study namely the meaning of gambling, beliefs about gambling, perception about themselves (i.e., gamblers) and cessation of gambling. Each structure contained inter-related themes that are co-dependent. The emerging structures or themes in this research are now examined in relation to past research.

This study found that the meaning of gambling to our participants was different from their beliefs about gambling. The meaning of gambling, which encompassed pleasure, excitement, meaning to life and recognition from family and friends, was described as the significance of the meaning of gambling behaviour to gamblers. Although participants were willing to change their habit of gambling, this did not change the meaning of gambling that they held onto. Gambling was seen as a source of entertainment, pleasure and excitement. Gamblers mentioned that if one cannot control themselves over the reward of winning and the pain of losing, they should not engage in gambling. However, they knew they could not successfully control their gambling urges so they tried their best not to think about past wins.

Some aspects of meaning of gambling to recovering gamblers remained the same even when they chose to quit gambling (e.g., gambling as a pleasurable hobby). However, participants found that spending time doing charity or community work with the Gamblers Rehabilitation Centre helped lower their preoccupation with gambling and gave them an altered positive sense of meaning in terms of contributing to the community. They also learned to see that monetary value is not as important as regaining the trust of their family, particularly their wives and children. This exemplifies a shift in finding meaning, dignity and recognition from gambling to finding meaning in positive contributions to significant others and community members.

Certain misconceptions about gambling seem more malleable than others. For example, some participants came to realize that there is no easy way to earn money; instead they were providing revenues to the gambling industry. During recovery, they acknowledged the unreliability of their belief about gambling as a form of investment with lucrative returns. As such, these recovering gamblers reported that they questioned their beliefs about gaining revenues from big wins during the recovery process. These findings may suggest that recovering gamblers may begin to view gambling as a game of chance and that there are other more viable avenues to gain financial income.

In addition, this study also discovered that the perception of self is closely linked with the gamblers' perceived perceptions of how their families viewed them. The participants defined themselves as "good" or "bad and selfish" with regards to their gambling habit and ability to provide monetary comfort and companionship to their family members. These findings are consistent with past research that has evidenced strained relationships due to gambling debts (Schwarz and Lindner, 1992; Vong, 2007). Participants felt proud and had a good self-image when they had the ability to gamble using their own money (i.e., not from borrowed funds) and provide for their families financially. Moreover, participants mentioned that they were committed caring fathers and husbands when they were not engaging in gambling activities. Family relationships and 
perceived connections are important factors that contribute to the gambling recovery process.

Evidently, some participants viewed themselves as self-centred individuals who do not care about others' wellbeing when they continued to gamble at the expense of their family's financial status. They also felt guilty for spending more time gambling and this guilt further escalated self-alienation from family members, which further progressed into neglecting responsibilities as a father, husband and son. Furthermore, some participants reported being disappointed with themselves when they relapsed into gambling behaviour after their family helped in settling debts.

Interestingly, this study found an emerging theme regarding family experiences of participants. Family experiences discussed earlier pointed out how family members or significant others of the gambler are affected by the gambling experience. The gamblers' self-perception has clearly been defined by their gambling habit in relation to how they were treating their family members or significant others. Thus, as mentioned in past research (Dickson-Swift et al., 2005; Corney and Davis, 2010; Valentine and Hughes, 2010), the family or significant others of the gambler do not only suffer from financial strains but may also be affected emotionally and psychologically. This is due to the inconsistency of the gamblers' behaviours and actions toward their family members and significant others. For example, when the gambler is perceived to be emulating his "good self," he will spend more time and money on his family. However, when he is perceived to be emulating his "bad self," he will cause turmoil and distress to his family members or significant others by neglecting them and increasing familial debt. Therefore, such inconsistencies in the gamblers' behaviours may add to the stress and cognitive dissonance that the family or significant others experience.

In this study, we found that a strong motivator for the participants' decision to quit gambling was related to their family's reaction towards their gambling habit and debts. Consistent with past research, this study found that most families were willing to assist the gambler in debt settlement during the initial stages but eventually ceased to help if the gambler persists in their gambling activities (Valentine and Hughes, 2010). In this study, most families had tried to assist in debt settlement approximately twice and some on up to five occasions, but some gamblers chose to continue gambling despite promising their families that they would stop. The turning-point and recovery occurred when the family took a firm stand in not assisting and not enabling gambling behaviour. In addition, these gamblers usually incurred a huge debt, which they were unable to settle. These were factors found in this study that influenced the participants' decision to seek treatment by joining a rehabilitation centre and to cease gambling.

According to past research (Kim, 2011; Loo et al., 2011), family support is an essential component of the recovery process and help-seeking behaviour. Our findings iterated the importance of family but it must be noted that social support such as encouragement and continuous acceptance is effective only after the gambler has decided to quit gambling based on their own free will. Financial support from the family while the gambler is still engaged in gambling activities may further escalate problem gambling as this monetary assistance is often viewed as a form of enabling behaviour. Family support plays a significant role in motivating gamblers to continue with recovery programs and abstain from gambling. Based on these findings, we are proponents of families being involved in the intervention and recovery process (Petry, 2005). 
In our findings, it is interesting to note that the meaning of gambling did not change for the recovering gambler as gambling abstinence is exhibited as a behavioural action that does not necessarily indicate that the recovering gambler has stopped finding meaning in gambling activities. As an implication for treatment providers, it is important for us to recognize this because it is essential that rehabilitation programs should focus on addressing gamblers' misconceptions (e.g., beliefs about gambling as a means to earn money) together with the meaning of gambling to the gamblers (i.e., gambling as a pleasurable hobby). These factors should be adequately addressed together and not in isolation. Alternatively, rehabilitation programs can assist gamblers in finding new associations to the meaning linked with gambling. This may help gamblers successfully change not only their gambling behaviour, but also their beliefs and personal meaning attributed to gambling.

Another implication that emerged is the impact of family members on gamblers' self-perception and decision to quit gambling. Gamblers' self-perception was linked to gambling wins and family's reaction to their gambling habit. Also, their choice to quit gambling was greatly influenced by their family's reaction and their perceived importance of family relationships. This finding may affect the family members and significant others related to the gambler. Therefore, families should be made aware of the power of their responses to the gambling habit as it can help the gamblers overcome their urge to gamble.

Another implication of this study involves rehabilitation centres. Some rehabilitation centres, particularly in Malaysia, do not allow contact with people outside their facility to ensure smooth running of the program and to prevent unwanted pressure from family members on the gamblers. However, our findings suggest that an incorporated family intervention system can be a highly positive influence on the recovery process. We suggest that rehabilitation centres include family members as part of the intervention process, particularly at a later stage of the rehabilitation program.

\section{Conclusion}

As this research has brought forth many themes that interact with the experiences of gamblers, there are various research avenues to further examine these themes. Familial influence plays an important role in the lives of gamblers as it was evidenced in this study that gamblers' perception about themselves are related to their families' responses towards their gambling habit. This study also found that the family members and significant others' reaction and management of the gambling issue influenced the gambler's decision to quit gambling. It will be interesting to discover more about the specific mechanisms involved in this recovery process.

In exploring gamblers' experiences, this study enhances a deeper understanding of the importance of family members and significant others in their decision to quit gambling and engage in the recovery process. It is clear that a strong family support system and restitution are essential components in any treatment plan.

Competing interests

The authors declare that they have no competing interests. 


\section{Acknowledgements}

All authors would like to thank Gamblers Rehabilitation Centre Malaysia for their assistance in the participant recruitment process.

\section{Author details}

${ }^{1}$ Department of Psychology, HELP University, Jalan Dungun, Kuala Lumpur 50490, Malaysia. ${ }^{2}$ Jeffrey Cheah School of Medicine and Health Sciences, Monash University Malaysia, Jalan Lagoon Selatan, Bandar Sunway 47500, Malaysia.

${ }^{3}$ Columbia Asia Hospital Seremban, Jalan Haruan 2, Oakland Commercial Center, Seremban 70300, Malaysia.

Received: 28 February 2013 Accepted: 8 January 2014

Published: 30 January 2014

\section{References}

American Psychiatric Association. (2000). Diagnostic and statistical manual of mental disorders: DSM-IV-TR (4th text revision). Washington, DC: American Psychiatric Association.

American Psychiatric Association. (2013). Diagnostic and statistical manual of mental disorders: DSM-5 (5th ed.). Washington, DC: American Psychiatric Association.

Bagby, RM, Vachon, DD, Bulmash, EL, Toneatto, T, Quilty, LC, \& Costa, PT. (2007). Pathological gambling and the five-factor model of personality. Personality and Individual Differences, 43(4), 873-880.

Baker, C, Wuest, J, \& Stern, PN. (1992). Method slurring: the grounded theory/phenomenology example. Journal of Advanced Nursing, 17(11), 1355-1360.

Basu, EO. (1991). Profit, loss, and fate: the entrepreneurial ethic and the practice of gambling in an overseas Chinese community. Modern China, 17(2), 227.

Blaszczynski, A, \& Nower, L. (2002). A pathways model of problem and pathological gambling. Addiction, 97, 487-499.

Breen, H, Hing, N, \& Gordon, A. (2013). Indigenous Australian gambling crime and possible interventions: a qualitative study. Asian Journal of Gambling Issues and Public Health, 3(1), 1-16.

Chan, CC, \& Ohtsuka, K. (2013). The clinical and social construction of the Paichais of Macau. Asian Journal of Gambling Issues and Public Health, 3(1), 1-11.

Ciarrocchi, JW, \& Reinert, DF. (1993). Family environment and length of recovery for married male members of Gamblers Anonymous and female members of GamAnon. Journal of Gambling Studies, 9(4), 341-352.

Ciarrocchi, JW, \& Richardson, R. (1989). Profile of compulsive gamblers in treatment: update and comparisons. Journal of Gambling Behavior, 5(1), 53-65.

Clarke, D, Tse, S, Abbott, M, Townsend, S, Kingi, P, \& Manaia, W. (2006). Key indicators of the transition from social to problem gambling. International Journal of Mental Health and Addiction, 4(3), 247-264.

Corney, R, \& Davis, J. (2010). Female frequent internet gamblers: a qualitative study investigating the role of family, social situation and work. Community, Work \& Family, 13(3), 291-309. doi: 10.1080/13668803.2010.488088.

Darbyshire, P, Oster, C, \& Carrig, H. (2001). The experience of pervasive loss: children and young people living in a family where parental gambling is a problem. Journal of Gambling Studies, 17(1), 23-45. doi: 10.1023/a:1014536315167.

Dickson-Swift, VA, James, EL, \& Kippen, S. (2005). The experience of living with a problem gambler: spouses and partners speak out. Journal of Gambling Issues(13). doi: 10.4309/jgi.2005.13.6.

Eisen, SA, Lin, N, Lyons, MJ, Scherrer, JF, Griffith, K, True, WR, \& Tsuang, MT. (1998). Familial influences on gambling behavior: an analysis of 3359 twin pairs. Addiction, 93(9), 1375-1384. doi: 10.1046/.1.1360-0443.1998.93913758.x.

Giorgi, A. (1989). Some theoretical and practical issues regarding the psychological phenomenological method. Saybrook Review, 7(2), 71-85.

Kim, W. (2011). Acculturation and gambling in Asian Americans: when culture meets availability. International Gambling Studies, 12(1), 69-88. doi: 10.1080/14459795.2011.616908.

Kim, JH, Lee, S, Chow, J, Lau, JTF, Tsang, A, Choi, J, \& Griffiths, SM. (2008). Prevalence and the factors associated with binge drinking, alcohol abuse, and alcohol dependence: a population-based study of Chinese adults in Hong Kong. Alcohol and Alcoholism, Vol 43(3), 360-370.

Lam, D. (2008). The brief Chinese history of gambling. Macau: University of Macau publications.

Lau, LY, \& Ranyard, R. (2005). Chinese and English probabilistic thinking and risk taking in gambling. Journal of Cross-Cultural Psychology, 36(5), 621-627.

Lee, HP, Chae, PK, Lee, HS, \& Kim, YK. (2007). The five-factor gambling motivation model. Psychiatry Research, 150(1), 21-32. doi: 10.1016/j.psychres.2006.04.005.

Loo, JMY, Raylu, N, \& Oei, TPS. (2008). Gambling among the Chinese: a comprehensive review. Clinical Psychology Review, 28(7), 1152-1166. doi: 10.1016/j.cpr.2008.04.001.7.

Loo, JMY, Oei, TPS, \& Raylu, N. (2011). Problem gambling, gambling correlates, and help-seeking attitudes in a Chinese sample: an empirical evaluation. Psychology, 2(4), 342-354.

Loo, JMY, Raylu, N, \& Oei, TPS. (2012). Testing the validity of an integrated cognitive behavioural model of gambling behaviour with a Chinese sample. In AE Cavanna (Ed.), Psychology of gambling: new research (pp. 119-137). New York: Nova Publishers.

Merriam, SB. (2002). Qualitative Research in Practice. New York: Jossey-Bass.

Moodie, C, \& Finnigan, F. (2006). Association of pathological gambling with depression in Scotland. Psychological Reports, 99(2), 407-417. doi: 10.2466/pro.99.6.407-417.

Nixon, G, \& Solowoniuk, J. (2006). An insider's look into the process of recovering from pathological gambling disorder: an existential phenomenological inquiry. International Journal of Mental Health and Addiction, 4(2), 119-132. doi: 10.1007/s11469-006-9012-1.

Nuske, E, \& Hing, N. (2012). A narrative analysis of help-seeking behaviour and critical change points for recovering problem gamblers: the power of storytelling. Australian Social Work, 66(1), 39-55. doi: 10.1080/ 0312407X.2012.715656. 
Oei, TPS, \& Raylu, N. (2004). Familial influence on offspring gambling: a cognitive mechanism for transmission of gambling behavior in families. Psychological Medicine, 34(7), 1279-1288.

Oei, TPS, Lin, J, \& Raylu, N. (2008). The relationship between gambling cognitions, psychological states, and gambling: a cross-cultural study of Chinese and Caucasians in Australia. Journal of Cross-Cultural Psychology, 39(2), 147-161. doi: $10.1177 / 0022022107312587$.

Ohtsuka, K, \& Ohtsuka, T. (2010). Vietnamese Australian gamblers' views on luck and winning: universal versus culture-specific schemas. Asian Journal of Gambling Issues and Public Health, 1(1), 34-46.

Papineau, E. (2001). Pathological gambling in the Chinese community, an anthropological viewpoint. Loisir \& Societe-Society and Leisure, 24(2), 557-582.

Papineau, E. (2013). The expansion of electronic gambling machines in China through anthropological and public health lenses. Asian Journal of Gambling Issues and Public Health, 3(1), 1-12.

Petry, NM. (2005). Therapy for families and significant others. In NM Petry (Ed.), Pathological gambling: etiology, comorbidity, and treatment (pp. 175-187). Washington, DC: American Psychological Association.

Raylu, N, \& Oei, TPS. (2002). Pathological gambling: a comprehensive review. Clinical Psychology Review, 22(7), 1009-1061.

Raylu, N, \& Oei, TPS. (2004a). The Gambling Urge Scale: development, confirmatory factor validation, and psychometric properties. Psychology of Addictive Behaviors, 18(2), 100-105

Raylu, N, \& Oei, TPS. (2004b). Role of culture in gambling and problem gambling. Clinical Psychology Review, 23(8), 1087-1114.

Raylu, N, Oei, TPS, \& Loo, JMY. (2008). The current status and future direction of self-help treatments for problem gamblers. Clinical Psychology Review, 28(8), 1372-1385. doi: 10.1016/j.cpr.2008.07.005.

Raylu, N, Loo, JMY, \& Oei, TPS. (2013). Treatment of gambling problems in Asia: Comprehensive review and implications for Asian problem gamblers. Journal of Cognitive Psychotherapy, 27(3), 297-322.

Rossi, PH. (1980). Why Families Move. London: SAGE Publications.

Scanlon, K, \& Whitehead, C. (2004). International Trends in Housing Tenure and Mortgage Finance. London: The Council of Mortgage Lenders.

Schwarz, J, \& Lindner, A. (1992). Inpatient treatment of male pathological gamblers in Germany. Journal of Gambling Studies, 8(1), 93-109.

Teo, P, Mythily, S, Anantha, S, \& Winslow, M. (2007). Demographic and clinical features of 150 pathological gamblers referred to a community addictions programme. Annals Academy of Medicine Singapore, 36(3), 165-168.

Thomas, AC, Sullivan, GB, \& Allen, FCL. (2009). A theoretical model of EGM problem gambling: more than a cognitive escape. International Journal of Mental Health and Addiction, 7(1), 97-107.

Turner, NE. (2008). Games, gambling, and gambling problems. In M Zangeneh, A Blaszczynski, \& NE Turner (Eds.), In the pursuit of winning: problem gambling theory, research and treatment (pp. 33-64). New York: Springer Science + Business Media.

Valentine, G, \& Hughes, K. (2010). Ripples in a pond: the disclosure to, and management of, problem internet gambling with/in the family. Community, Work \& Family, 13(3), 273-290. doi: 10.1080/13668803.2010.488107.

Volberg, RA, Nysse-Carris, KL, \& Gerstein, DR. (2006). 2006 California problem gambling prevalence survey. Los Angeles: California Department of Alcohol and Drug Programs Office of Problem and Pathological Gambling.

Vong, F. (2007). The psychology of risk-taking in gambling among Chinese visitors to Macau. International Gambling Studies, 7(1), 29-42.

Wardle, H, Sproston, K, Orford, J, Erens, R, Griffiths, M, Constantine, R, et al. (2007). British gambling prevalence survey 2007. London: National Centre for Social Research.

Wolfling, K, Morsen, CP, Duven, E, Albrecht, U, Grusser, SM, \& Flor, H. (2011). To gamble or not to gamble: at risk for craving and relapse - learned motivated attention in pathological gambling. Biological Psychology, 87(2), 275-281. doi: 10.1016/j.biopsycho.2011.03.010.

doi:10.1186/2195-3007-4-

Cite this article as: Choong et al: The experience of recovering gamblers in Malaysia: a phenomenological study. Asian Journal of Gambling Issues and Public Health 2014 4:1.

\section{Submit your manuscript to a SpringerOpen ${ }^{\circ}$ journal and benefit from:}

- Convenient online submission

- Rigorous peer review

- Immediate publication on acceptance

- Open access: articles freely available online

- High visibility within the field

- Retaining the copyright to your article

Submit your next manuscript at $\boldsymbol{\sim}$ springeropen.com 